\title{
A Dual Selection Marker Transformation System Using Agrobacterium tumefaciens for the Industrial Aspergillus oryzae 3.042 ${ }^{\mathrm{S}}$
}

\author{
Yunlong Sun ${ }^{1}$, Yali Niu ${ }^{1}$, Bin $\mathrm{He}^{1}$, Long Ma ${ }^{1}$, Ganghua $\mathrm{Li}^{2}$, Van-Tuan $\mathrm{Tran}^{3,4}$, Bin Zeng ${ }^{1 *}$, and Zhihong $\mathrm{Hu}^{1 *}$ \\ ${ }^{1}$ Jiangxi Key Laboratory of Bioprocess Engineering and Co-Innovation Center for In-vitro Diagnostic Reagents and Devices of Jiangxi \\ Province, College of Life Sciences, Jiangxi Science E Technology Normal University, Nanchang 330013, P.R. China \\ ${ }^{2}$ Hubei Key Laboratory of Edible Wild Plants Conservation and Utilization (Hubei Normal University), 435002, Huangshi, P.R. China \\ ${ }^{3}$ National Key Laboratory of Enzyme and Protein Technology, VNU University of Science, 334 Nguyen Trai, Thanh Xuan, Hanoi, Vietnam \\ ${ }^{4}$ VNU University of Science, 334 Nguyen Trai, Thanh Xuan, Hanoi, Vietnam
}

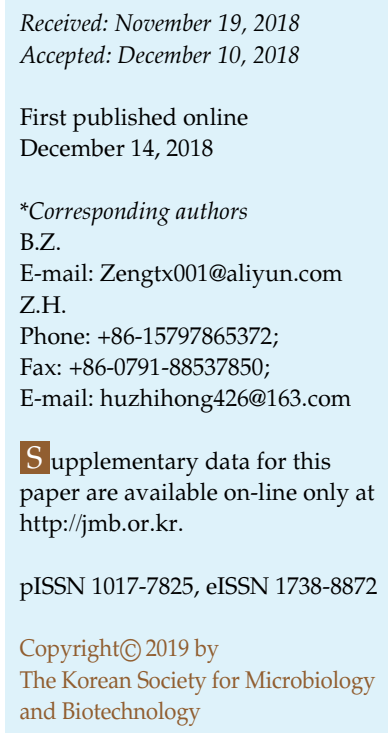

Currently, the genetic modification of Aspergillus oryzae is mainly dependent on protoplastmediated transformation (PMT). In this study, we established a dual selection marker system in an industrial A. oryzae 3.042 strain by using Agrobacterium tumefaciens-mediated transformation (ATMT). We first constructed a uridine/uracil auxotrophic A. oryzae 3.042 strain and a pyrithiamine (PT)-resistance binary vector. Then, we established the ATMT system by using uridine/uracil auxotrophy and PT-resistance genes as selection markers. Finally, a dual selection marker ATMT system was developed. This study demonstrates a useful dual selection marker transformation system for genetic manipulations of $A$. oryzae 3.042 .

Keywords: Aspergillus oryzae, uridine/uracil auxotrophy, pyrithiamine, Agrobacterium tumefaciensmediated transformation
Aspergillus oryzae has a long history in traditional food fermentation, condiment production, and brewing industries in Asia [1, 2]. Its industrial popularity has increased over recent years, particularly for recombinant protein production, where an increasing number of researchers have utilized genetic engineering techniques to improve fermentation performance and product yield [3, 4]. Selection markers and transformation methods are key considerations for genetic manipulations. Since $A$. oryzae shows inherent resistance to common antibiotics $[5,6]$, auxotrophic genes are often used as selection markers [7-12]. PT is a vitamin B1 antagonist, and the PT resistance gene-ptrA which has been cloned from a PT-resistant $A$. oryzae mutant can also be used as a selection marker [13, 14]. PMT and ATMT are the main methods used for transforming filamentous fungi [15]. Compared with PMT, ATMT is easier to conduct and the transformation efficiency is high $[15,16]$. Attempts to establish an ATMT system in A. oryzae were unsuccessful until 2016, when Nguyen et al. (2016) established ATMT in A. oryzae using uracil auxotrophy as a selectable marker [17]. Herein, we constructed a dual selection marker transformation system using the ATMT method in an industrial $A$. oryzae 3.042 strain.

Our sensitivity tests suggested A. oryzae 3.042 is sensitive to 5-FOA (Fig. S1A). Therefore, 5-FOA was to be used for construction of the uridine/uracil auxotrophy mutant, as in a previous study [18]. The binary vector pAoG (supplementary materials), was used to delete the $p y r G$ gene (uridine/uracil auxotrophy gene) in A. oryzae 3.042. Transformation of pAoG was performed using a method described by Nguyen et al. (2017) with minor revisions (supplementary materials). Five transformant colonies were selected for phenotypic and PCR identification (the primer sequences are listed in Table S1). Results suggested 
A

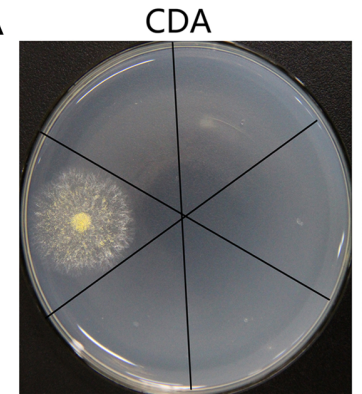

B

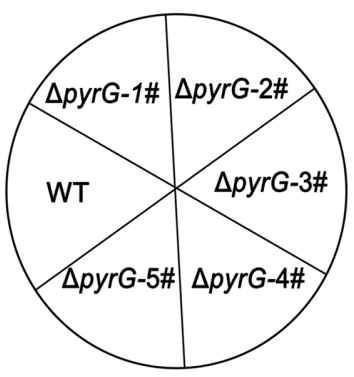

$\mathrm{CDA}+\mathrm{Ura}+\mathrm{Uri}$

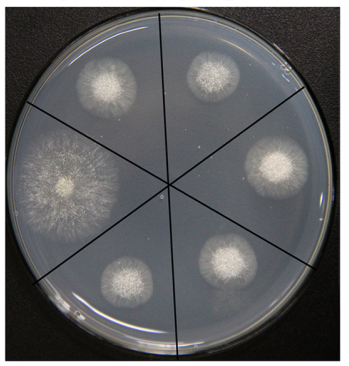

C
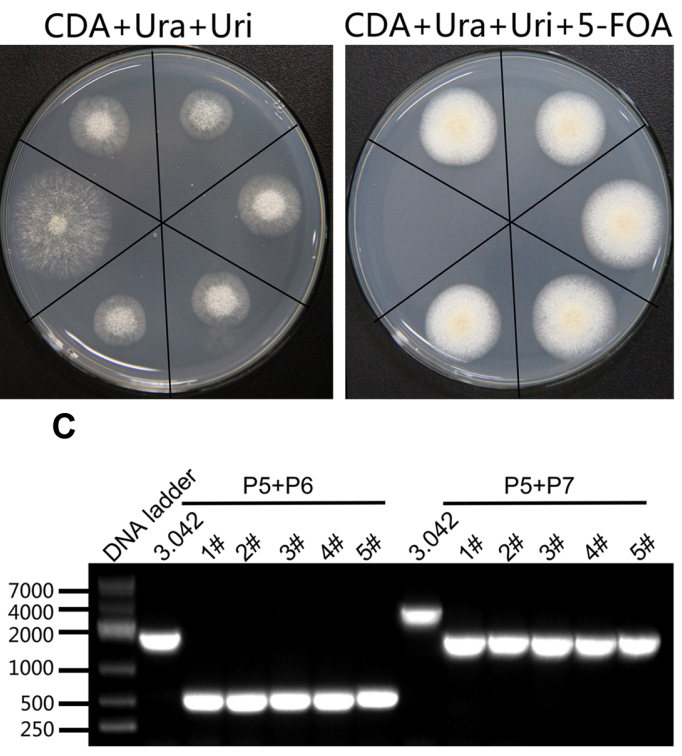

Fig. 1. The deletion of the $p y r G$ gene in Aspergillus oryzae 3.042.

(A) The transformants obtained from the 5-FOA medium were confirmed on the CDA, CDA + uridine + uracil, and CDA + uridine + uracil + 5FOA media. (B) The scheme of the different $A$. oryzae strain cultures in (A). (C) The $p y r G$ deletion mutants were confirmed by PCR analysis using two different primer pairs. 1\# to $5 \#$ represent the different colonies.

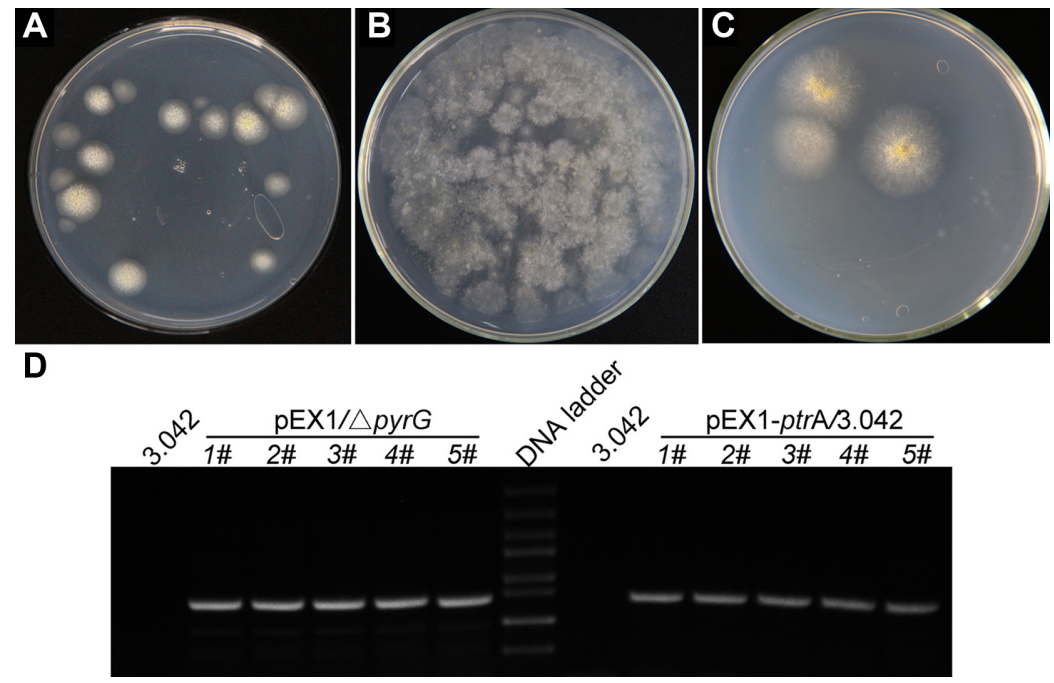

Fig. 2. The identification of pEX1 and pEX1-ptrA transformants.

(A) Plate showing pEX1 transformation after cultivation for 5 days on the screening medium. (B) Plate showing pEX1-ptrA transformation $60 \mathrm{~h}$ after cultivation for 3 days on the screening medium. (C) Plate showing pEX1-ptrA transformation $48 \mathrm{~h}$ after cultivation for 5 days on the screening media. (D) The transformants were confirmed by PCR using the specific primer pairs for the GFP reporter gene. The co-cultivation concentration of spores was $10^{5} / \mathrm{ml}$.

the $\operatorname{pyrG}$ was successfully knocked out (Figs. 1A and 1B).

Sensitivity tests revealed A. oryzae 3.042 is as sensitive to PT as other strains (Fig. S1A). Therefore, we replaced the pyrG cassette of pEX1 (the plasmid used for ATMT in A. oryzae RIB40 [17]) with a ptrA cassette to construct the pEX1-ptrA vector (Fig. S1B). The pEX1 and pEX1-ptrA constructs were transformed into A. oryzae $3.042 \Delta p y r G$ and A. oryzae wild type. For the pEX1 construct, an average of 
10 transformants per plate were obtained; the transformation efficiency was over 100 transformants per $10^{5}$ spores (Fig. 2A). For the pEX1-ptrA construct, background growth was strong, which complicated the selection of positive transformants (Fig. 2B). This may be because PT cannot inhibit hyphae growth, which occurred in some spores that had germinated during co-cultivation. Consequently, co-cultivation time was decreased from $60 \mathrm{~h}$ to $48 \mathrm{~h}$ to avoid spore germination, thereby decreasing background growth. The resulting transformants were easier to select, with an efficiency of approximately 30 transformants per $10^{5}$ spores (Fig. 2C). Transformed colonies were confirmed by the amplification of the GFP reporter gene (Fig. 2D).

Transformants were cultured on the corresponding selective media to examine their growth phenotype. Results indicated that the pEX1 transformants complemented the uridine/uracil auxotrophy and the pEX1-ptrA transformants demonstrate PT-resistance (Figs. 3A and 3B). Then, the GFP fluorescence was observed, and it was found that both pEX1 and pEX1-ptrA transformants can successfully express the GFP reporter gene (Figs. $3 \mathrm{C}-3 \mathrm{H}$ ). Therefore, the $\mathrm{pEX} 1$ and pEX1-ptrA vectors can be employed for the transformation of $A$. oryzae 3.042 via the ATMT method.

Finally, a dual selection marker transformation system in A. oryzae 3.042 was established. Since both pEX1 and pEX1ptrA encode GFP, we selected $\mathrm{pEX} 2 \mathrm{~B}$, an alternative binary vector with a uridine/uracil auxotrophy selection marker and DsRed reporter gene [9]. We used the pEX1-ptrA and pEX2B vector to construct a dual selection marker transformation system. The pEX1-ptrA was transformed into A. oryzae 3.042 spyrG; then, the transformant was used as the host to transform with $\mathrm{pEX} 2 \mathrm{~B}$. The dual transformant was screened for both selection markers. Fluorescence of the GFP and DsRed were observed, confirming successful transformation. As shown in Fig. 4, the pEX1-ptrA/A. oryzae 3.042 spyrG and pEX2B/A. oryzae 3.042 spyrG strains display either GFP or DsRed fluorescence, respectively; while in dual transformants, both GFP and DsRed fluorescence were observed (Fig. 4). Thus, our studies revealed that uridine/uracil auxotrophy and PT-resistance can be used as a dual selection marker for ATMT in A. oryzae 3.042 .

In summary, this study established a novel two-marker
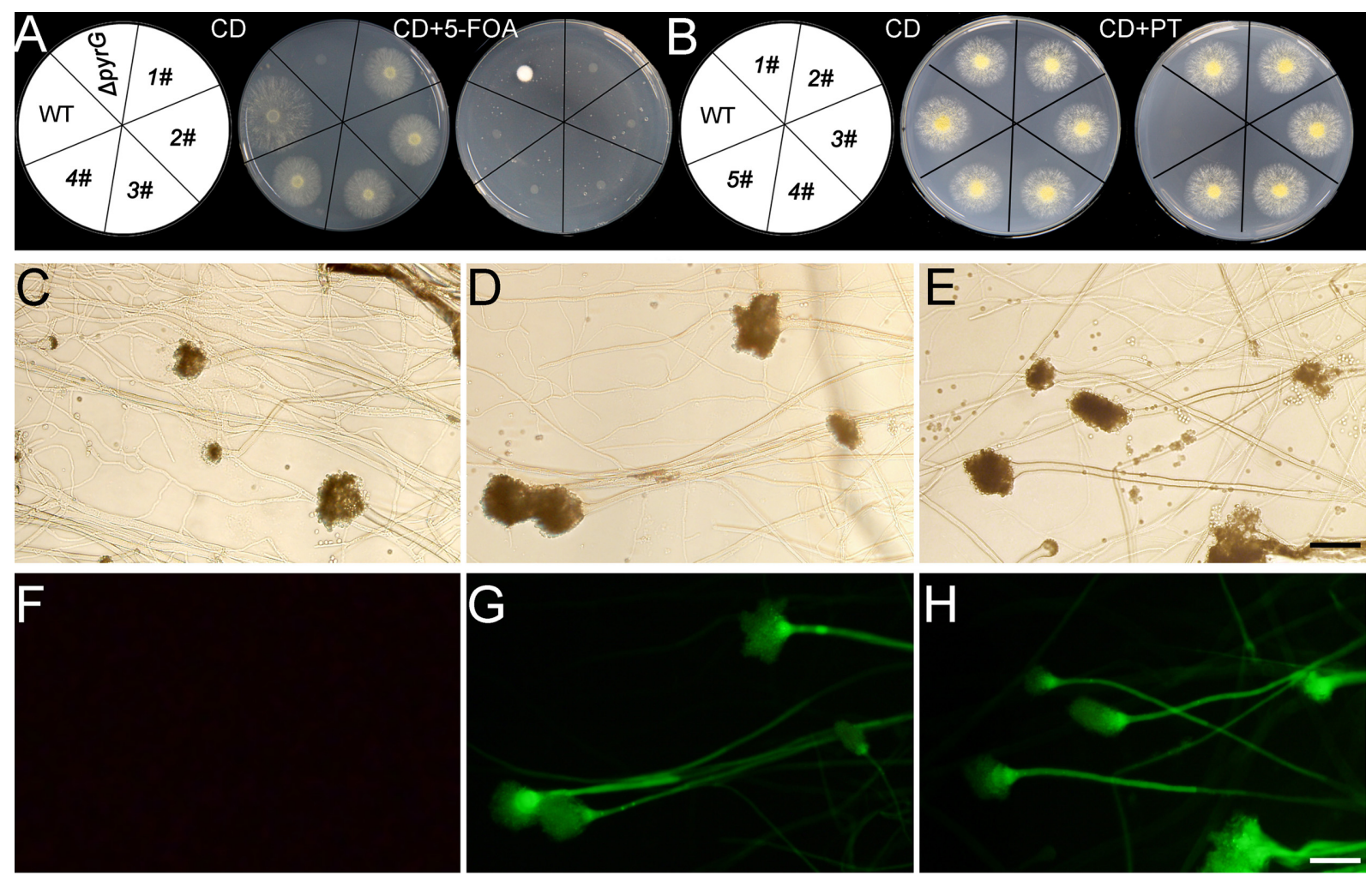

Fig. 3. Confirmation of transformants by assaying their growth on selective media and GFP observation.

(A and B) The growth of pEX1/A. oryzae $3.042 \Delta p y r G$ and pEX1-ptrA/A. oryzae 3.042 on different media; (C-E) and (F-H) The 3-day-old mycelia of A. oryzae 3.042, pEX1/A. oryzae $3.042 \Delta p y r G$, and pEX1-ptrA/A. oryzae 3.042 under bright field and fluorescent field microscopy. 


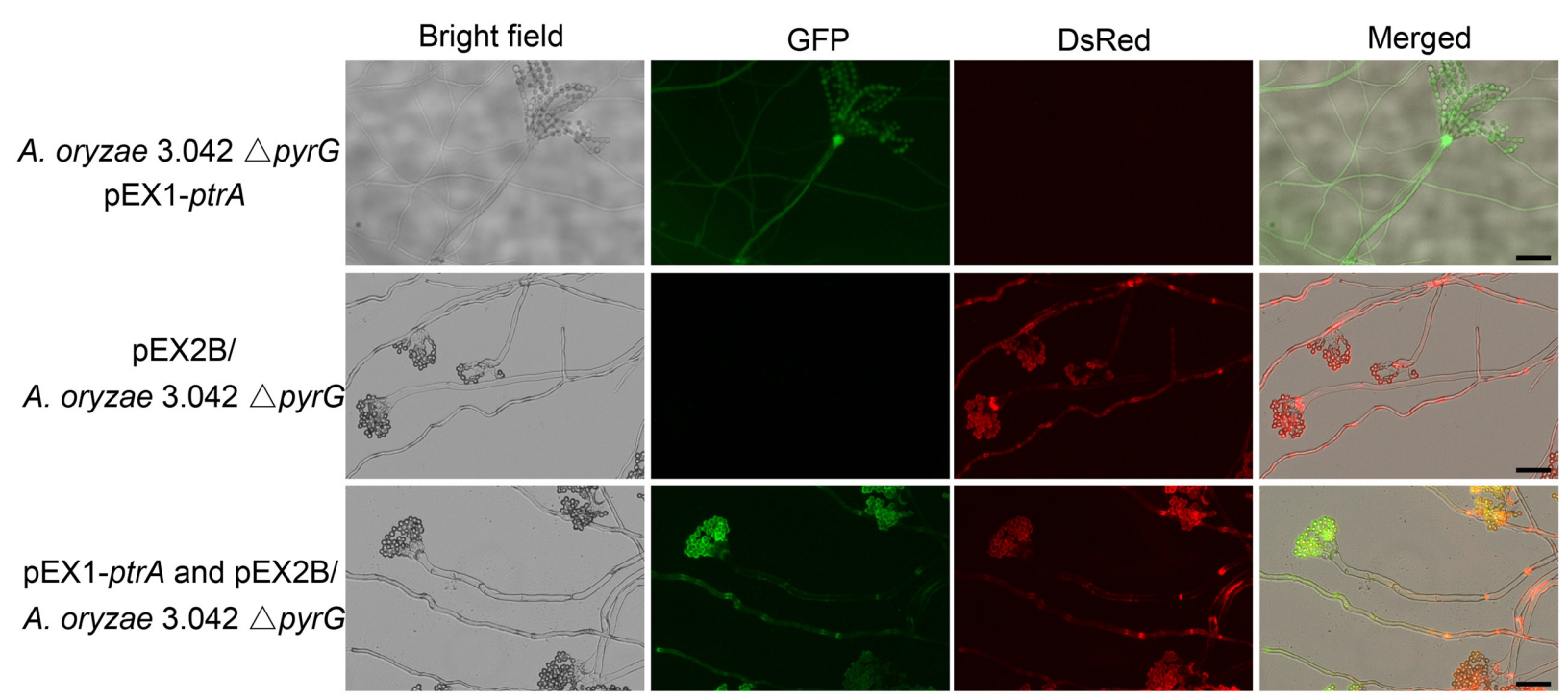

Fig. 4. GFP and DsRed fluorescence in the transformants.

The mycelia of transformants were grown under coverslips for 3 days. Only GFP (Second column) or DsRed (third column) can be observed in pEX1-ptrA/A. oryzae $3.042 \Delta p y r G$ or pEX2B/A. oryzae 3.042 ApyrG transformants, respectively; both GFP and DsRed can be observed in the dual transformants (fourth column).

selection system in an A. oryzae 3.042 strain. For the first time we demonstrate the use of ATMT to transform PTresistance as a selection marker in A. oryzae. An advantage of ATMT using PT-resistance as a selectable marker in ATMT is that it can be performed on the wild-type A. oryzae. However, selection efficiency was less than when using the uridine/uracil auxotrophy method. The possible reasons are as follows: firstly, although PT showed strong inhibition of $A$. oryzae spore germination, it cannot inhibit mycelia growth once the spores germinated [13]; secondly, the presence of a very small amount of vitamin B1 in the culture system (such as the tissue fluid from lysing cells or vitamin B1 in culture medium) also greatly reduces the inhibitory effect [13]. Despite this, we believe our study shows the establishment of a dual selection marker transformation system using ATMT, which can simplify transformation procedures and improve the efficiency of genetic manipulations in A. oryzae. This could facilitate functional gene research, which can improve the fermentation performance and productivity of A. oryzae.

\section{Acknowledgments}

This study was supported by the National Natural Science Foundation of China (NSFC Grant Nos: 31700068 and 31460447), Natural Science Foundation of Jiangxi Province (Grant Nos: 20181BAB214001 and 20171BAB214004), the
Open Foundation of Hubei Key Laboratory of Edible Wild Plants Conservation and Utilization (Grant No: EWPL201705) and Research Project of Jiangxi Provincial Department of Education (Grant No:GJJ180600).

\section{Conflict of Interest}

The authors have no financial conflicts of interest to declare.

\section{References}

1. Yamada R, Yoshie T, Wakai S, Asai-Nakashima N, Okazaki F, Ogino C. et al. 2014. Aspergillus oryzae-based cell factory for direct kojic acid production from cellulose. Microb. Cell Fact. 13: 71 .

2. Kitamoto K. 2015. Cell biology of the Koji mold Aspergillus oryzae. Biosci. Biotechnol. Biochem. 79: 863-869.

3. Wang B, Guo G, Wang C, Lin Y, Wang X, Zhao M, et al. 2010. Survey of the transcriptome of Aspergillus oryzae via massively parallel mRNA sequencing. Nucleic. Acids Res. 38: 5075-5087.

4. Ichishima E. 2016. Development of enzyme technology for Aspergillus oryzae, A. sojae, and A. luchuensis, the national microorganisms of Japan. Biosci. Biotechnol. Biochem. 80: 1681-1692.

5. Suzuki S, Tada S, Fukuoka M, Taketani H, Tsukakoshi Y, Matsushita M, et al. 2009. A novel transformation system 
using a bleomycin resistance marker with chemosensitizers for Aspergillus oryzae. Biochem. Biophys. Res. Commun. 383: 42-47.

6. Gomi K., Kitamoto, K., \& Kumagai, C. 1992. Transformation of the industrial strain of Aspergillus oryzae, with the homologous amds, gene as a dominant selectable marker. J. Ferment. Bioeng. 74: 389-391.

7. Lubertozzi D, Keasling JD. 2006. Marker and promoter effects on heterologous expression in Aspergillus nidulans. Appl. Microbiol. Biotechnol. 72: 1014-1023.

8. Ling SOS, Storms R, Zheng Y, Rodzi MRM, Mahadi NM, Illias RM, et al. 2013. Development of a pyrG mutant of Aspergillus oryzae strain S1 as a host for the production of heterologous proteins. Scientific World J. 2013: 1-7.

9. Nguyen KT, Ho QN, Do L, Mai LTD, Pham DN, Tran HTT, et al. 2017. A new and efficient approach for construction of uridine/uracil auxotrophic mutants in the filamentous fungus Aspergillus oryzae using Agrobacterium tumefaciensmediated transformation. World J. Microbiol. Biotechnol. 33: 107.

10. Jin FJ, Maruyama J, Juvvadi PR, Arioka M, Kitamoto K. 2004. Development of a novel quadruple auxotrophic host transformation system by $\arg B$ gene disruption using adeA gene and exploiting adenine auxotrophy in Aspergillus oryzae. FEMS Microbiol. Lett. 239: 79-85.

11. Jin FJ, Maruyama J, Juvvadi PR, Arioka M, Kitamoto K. 2004. Adenine auxotrophic mutants of aspergillus oryzae: development of a novel transformation system with triple auxotrophic hosts. Biosci. Biotechnol. Biochem. 68: 656-662.

12. Du Y, Xie G, Yang C, Fang B, Chen H. 2014. Construction of brewing-wine Aspergillus oryzae pyrG- mutant by pyrG gene deletion and its application in homology transformation. Acta Biochim. Biophys. Sin. 46: 477-483.

13. Kubodera T, Yamashita N, Nishimura A. 2000. Pyrithiamine resistance gene $(p \operatorname{tr} A)$ of Aspergillus oryzae: cloning, characterization and application as a dominant selectable marker for transformation. Biosci. Biotechnol. Biochem. 64: 1416-1421.

14. Kubodera T, Yamashita N, Nishimura A. 2002. Transformation of Aspergillus sp. and Trichoderma reesei using the pyrithiamine resistance gene (ptrA) of Aspergillus oryzae. Biosci. Biotechnol. Biochem. 66: 404-406.

15. Weyda I, Yang L, Vang J, Ahring BK, Lübeck M, Lübeck PS. 2017. A comparison of Agrobacterium-mediated transformation and protoplast-mediated transformation with crispr-cas9 and bipartite gene targeting substrates, as effective gene targeting tools for Aspergillus carbonarius. J. Microbiol. Methods 135: 26-34.

16. Idnurm A, Bailey AM, Cairns TC, Elliott CE, Foster GD, Ianiri $G$, et al. 2017. A silver bullet in a golden age of functional genomics: the impact of Agrobacterium-mediated transformation of fungi. Fungal Biol. Biotechnol. 4: 6.

17. Nguyen KT, Ho QN, Pham TH, Phan TN, Tran VT. 2016. The construction and use of versatile binary vectors carrying pyrG auxotrophic marker and fluorescent reporter genes for Agrobacterium-mediated transformation of Aspergillus oryzae. World J. Microbiol. Biotechnol. 32: 204.

18. Ling SO, Storms R, Zheng Y, Rodzi MR, Mahadi NM, Illias RM, et al. 2013. Development of a pyrG mutant of Aspergillus oryzae strain S1 as a host for the production of heterologous proteins. Scientific World J. 2013: 634317. 